\title{
Kontrollgruppe Schweizer Ärzte
}

\section{Yvonne Gilli}

Dr. med., Mitglied des FMH-Zentralvorstandes, Departementsverantwortliche Digitalisierung/eHealth

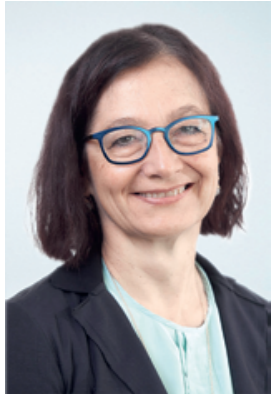

Die im Jahr 2015 publizierte SISA-Studie zeichnet ein eher düsteres Bild der Digitalisierung innerhalb der ambulant tätigen Ärzteschaft [1]. Dies obwohl der Grad der Digitalisierung in Schweizer Arztpraxen gemäss der jährlichen OECD-Befragung stetig zunimmt. Der Anteil der Ärztinnen und Ärzte, die elektronische Krankengeschichten führen, ist im Jahr 2018 auf 50\% angestiegen [2]. Die SISA-Studie zeigt aber auch, dass die Schweizer Ärzte Teil eines globalen Experiments sind: Das Schweizer Gesundheitssystem, in seiner Organisation und unter dem Aspekt der marktwirtschaftlichen Prinzipien vergleichbar mit dem amerikanischen oder kanadischen System, kennt im Gegensatz zu diesen keine entsprechenden Anreize, um die

\section{«Digitalisierung bedeutet weit mehr als das elektronische Patientendossier.»}

Akzeptanz und Nutzung von IT-Lösungen in der Gesundheitsversorgung zu verbessern oder eHealth $\mathrm{zu}$ fördern. Die Schweizer Ärzteschaft übernimmt also gewissermassen die Rolle einer Kontrollgruppe, welche die Digitalisierung vorantreibt, und zwar ohne ein staatliches Anreizsystem. Die Ergebnisse einer FMHStudie über die Digitalisierung der medizinischen Versorgung aus Ärztesicht (publiziert in dieser Ausgabe, Seiten 1685-1689) attestiert der Ärzteschaft eine positive Grundeinschätzung und Aufgeschlossenheit gegenüber digitalen Gesundheitsangeboten. Die Studie der FMH hat unmissverständlich vergegenwärtigt, dass die Bedenken der Ärzteschaft in Bezug auf die Digitalisierung ernst genommen werden müssen und ihr Einbezug für den Erfolg der Umsetzung von eHealth in der Schweiz entscheidend ist. Gleichzeitig müssen im Bereich eHealth, und damit ist selbstredend die Praxisinformatik eingeschlossen, die Systeminteroperabilität als notwendige Voraussetzung einer durchgängigen Prozessdigitalisierung gefördert sowie Anreize zur Digitalisierung gesetzt werden.

Das Departement Digitalisierung/eHealth hat dieses Jahr die Arbeiten im Bereich der Praxisinformatik aufgenommen, welche das Institut für Praxisinformatik dankenswerterweise initiierte. Vor wenigen Wochen hat das Departement die Tagung «Forum Praxisinformatik» durchgeführt mit dem Ziel, die Umsetzung der Digitalisierung anhand konkreter Fallbeispiele in der Arztpraxis aufzuzeigen und den Austausch der Ärzteschaft und der Industrie zu fördern. Präsentationen und Diskurs haben gezeigt, dass Digitalisierung weit mehr bedeutete als das elektronische Patientendossier. Die Umsetzung digitaler Prozesse in der Arztpraxis scheitert oftmals an scheinbar banalen Dingen, zum Beispiel der Durchgängigkeit von Daten zwischen Anwendungen in verschiedenen Bereichen wie Buchhaltung, Bestellwesen oder Qualitätsmanagement. Fehlende Integration und Interoperabilität führen sehr schnell zu einer Disruption der Arbeitsabläufe in der Arztpraxis, und offenbar stehen wir immer noch am Anfang der Problemlösung: Die «Usability» von medizinischen Informationssystemen hat sich in den letzten Jahren kaum verändert, wie eine wiederkehrende nationale Umfrage aus Finnland zeigt [3].

Fehlende Anreize haben auch zu einer geringen Verbreitung des Dossier Médical Partagé (DMP) in Frankreich geführt [4]. 5 Jahre nach Start des DMP haben lediglich 1,5\% der Bevölkerung ein DMP eröffnet, und weniger als 1\% der Ärztinnen und Ärzte im privaten Sektor beteiligt sich am DMP. Gründe auf ärztlicher Seite lagen in den Investitionskosten für ein System

Für eine erfolgreiche Umsetzung von eHealth ist der Einbezug der Ärzteschaft und die Entwicklung eines Anreizsystems entscheidend.

mit DMP-Schnittstellen. Erst im zweiten Anlauf wurde das Programm grundlegend modifiziert und ein Anreizsystem entwickelt. Die Anzahl der Dossiers sind in den ersten 10 Monaten nach Start des neuen DMP um $50 \%$ gestiegen. Bleibt zu hoffen, dass die Schweizer Ärzte von der Kontrollgruppe in die Interventionsgruppe wechseln.

\section{Literatur}

1 Djalali S, Ursprung N, Rosemann T, Senn O, Tandjung R. Undirected health IT implementation in ambulatory care favors paper-based workarounds and limits health data exchange. Int J Med Inform. 2015.

2 Golder L, Jans C. Swiss eHealth Barometer 2018, 2018.

3 Kaipio J, et al. Usability problems do not heal by themselves: National survey on physicians' experiences with EHRs in Finland. nt J Med Inform. 2017

4 Séroussi B, Bouaud J. Adoption of a Nationwide Shared Medical Record in France: Lessons Learnt after 5 Years of Deployment. Amin Annu Symp Proc Arch. 2016. 